\title{
Presentazione di una nuova Rubrica: la Nefrologia Narrativa
}

\author{
Francesco Logias ${ }^{1}$, Giuseppe Vanacore ${ }^{2}$, Marco Lombardi ${ }^{3}$ \\ ${ }^{1}$ Direttore U.O.C. di Nefrologia e Dialisi, P.O. San Francesco Nuoro ATS Sardegna, ASSL Nuoro, Nuoro \\ ${ }^{2}$ Presidente dell'Associazione Nazionale Emodializzati - Dialisi e Trapianto Onlus, Milano \\ ${ }^{3}$ Editor in Chief, Giornale di Tecniche Nefrologiche e Dialitiche, Firenze
}

Con il 2017 il Giornale si arricchisce di una nuova rubrica ma soprattutto di una nuova, importante e molto sentita collaborazione, quella con ANED. Insieme, le figure referenti della Nefrologia Narrativa, collaboreranno per tenere una Rubrica dedicata ai pazienti, alla quale potranno contribuire medici, psicologi, infermieri, familiari e amici, che potranno raccontare le loro storie di vita. In questa Rubrica coordinata dal Dottor Francesco Logias e dal Presidente di ANED Dottor Giuseppe Vanacore sono coinvolti alcuni illustri e noti nefrologi, personale di ANED, infermieri, pazienti e familiari di pazienti, di seguito elencati in puro ordine alfabetico: Patrizia Babini, Ornella Castelnovo, Alessandro Carrus, Carlo Maria Guastoni, Marco Minali, Giorgina Barbara Piccoli, Barbara Pittau, Antonio Santoro e Mario Timio.

La medicina narrativa nasce alla fine degli anni Novanta del Novecento nella Harvard Medical School di Boston, grazie a due psichiatri e antropologi: Arthur Kleinman e Byron Good. Altro pilastro fondatore è Rita Charon che, quasi contemporaneamente ai due psichiatri, ha sviluppato un programma di medicina narrativa presso la Columbia Medical School di New York, e ha avviato poi il primo corso di laurea nel 2009.

Byron Good scrive che "la narrazione è una forma in cui l'esperienza viene raccontata e rappresentata, insieme agli eventi vissuti dalle persone coinvolte che danno un senso alle loro storie".

La medicina narrativa è un momento di aggregazione, può essere considerata uno strumento per interpretare i disagi soggettivi e programmare interventi finalizzati, e favorisce un dialogo tra il medico e il paziente con effetti terapeutici.

Accepted: February 10, 2017

Published online: February 24, 2017

Indirizzo per la corrispondenza:

Dr. Marco Lombardi

Editor in Chief del Giornale di Tecniche Nefrologiche e Dialitiche

Firenze

lombardim@tin.it
Secondo Lisa Sanders, medico ma anche scrittrice, "nelle facoltà di medicina si ripete continuamente ai futuri medici che se ascolteranno il paziente, questi dirà loro cos'ha".

Nella nostra vita scolastica ognuno di noi, per tanti anni (spesso dalle elementari alla maturità), ha scritto racconti o componimenti. Abbiamo raccontato di noi, dei nostri genitori, degli amici, dei vicini di casa, ma anche di sconosciuti, di luoghi e tempi, di animali e cose. Abbiamo raccontato avvenimenti appena vissuti, oppure episodi del nostro passato, o le nostre aspettative per il futuro.

Raccontare il nostro vissuto ha una funzione catartica, in quanto la sofferenza viene inserita in racconti reali e diventa accettabile, si trasforma in risorsa.

La narrativa compare sulla scena proprio nell' istante in cui la medicina sembra perdere la sua efficacia nel rapporto con il paziente, e di conseguenza nell'individuazione e gestione degli stati di sofferenza.

Eugenia Tognotti docente dell'università di Sassari sostiene che "la crisi della medicina ad alta specializzazione tecnologica non ha fatto che aumentare la distanza fra medico e malato: dai trenta centimetri di stetoscopio che nell' 800 hanno cominciato a separare l'orecchio del medico dal corpo del paziente fino alle macchine sempre più sofisticate che, pur permettendo gli straordinari progressi della medicina, hanno però dilatato ulteriormente questa distanza".

Perciò i medici di oggi, oltre al sapere scientifico, devono sviluppare le proprie capacità di ascoltare e comprendere immediatamente i processi psichici del paziente. La medicina narrativa si può applicare in vari campi: I'anamnesi vitale e di relazione del vissuto del paziente, la condivisione della malattia, I'integrazione di altre discipline quali la sociologia, la psicologia, l'antropologia, da sempre parti integranti dello scibile medico.

Quando a Rita Charon, durante un'intervista, fu chiesto cosa la spinse a introdurre la medicina narrativa nel corso di studi, come se fosse un'altra specializzazione, lei rispose: "i malati hanno bisogno di medici che capiscano il loro star male, ascoltino i loro problemi e li accompagnino attraverso la loro malattia: al progresso tecnologico e diagnostico non 
ha fatto seguito una altrettanto avanzata ricerca sull'empatia, sul coraggio e sull'onestà per affiancare i pazienti nel viaggio dentro la loro malattia".

L'esperienza nella medicina narrativa è indispensabile per ascoltare, ghermire e rispettare significati e convinzioni personali, per interpretare e rispondere con altri mezzi e non solo con la terapia farmacologica. La medicina narrativa trascina la riflessione, presuppone professionalità specifica e crea un rapporto di fiducia, quel collegamento tra medico e paziente senza il quale nessuna terapia può essere accettata in modo efficace.

Sempre la Charon afferma: "Forse per noi qui è stato più facile, perché alla Columbia c'è sempre stata familiarità e interscambio con il mondo della filosofia, della letteratura e dell'antropologia, perché gli studiosi di queste discipline per primi volevano capire che strano mondo di relazioni esiste in ospedale, dove i ruoli sono definiti ma sospesi nello stesso tempo, dove i malati sono diversi da come erano fuori e si consegnano nelle mani di qualcun altro come non avevano mai fatto prima; già studiavano i rapporti in essere e le loro conseguenze in termini di terapia".

Di fatto i medici si sono ritrovati a leggere e studiare grandi scrittori e poi a immedesimarsi nei racconti sulla loro malattia. II racconto offre l'occasione di inserire concetti clinici e soprattutto opportunità che permettono di leggere la storia con gli occhi degli altri, originando una ricchezza e una pluralità di prospettive. La narrativa consente al paziente di sentirsi non isolato, ma al centro della struttura di salute e offre, a sua volta, ai medici e agli altri operatori sanitari la possibilità di avere una visione diversa.

Con la narrazione il paziente non è ridotto $\mathrm{a}$ un organo $\mathrm{o} a$ un apparato da curare ma rimane persona, individuo, portatore di un disagio che penalizza il suo vivere. La Dottoressa Franca Pellini, fondatrice di ANED, sostenuta dalla sua straordinaria sensibilità, intuì precocemente l'importanza per il paziente di partecipare attivamente al percorso di cura e di raccontarsi per diventare protagonista diretto della propria malattia.

La medicina narrativa è un nuovo modello che serve a far riflettere sulla nostra disciplina, per inseguire e ottenere fiducia, per sviluppare quella capacità di comprendere le sofferenze e le attese di un'altra persona, che anche la letteratura scientifica riconosce come elemento essenziale per il successo delle terapie, anche nelle situazioni più complesse come le malattie croniche.

La medicina narrativa diventa un'azione terapeutica che ha bisogno di mettersi in mostra nel mondo della letteratura, della pittura, della musica, del cinema e teatro, e di tutte quelle forme espressive che aiutano a ricomporre gli effetti e le frantumazioni dolorose di una patologia grave e invalidante, e il rapporto con il mondo esterno. 